\title{
Assessment of Left Atrial Function after ST-Segment Elevation Acute Myocardial Infarction: An Application of Real Time Three-Dimensional Echocardiography
}

yuxia $\mathrm{Miao}^{1}$, min $\mathrm{Xu}^{1}$, yuetao $\mathrm{Wang}^{1}$, xiao Xie ${ }^{1}$, fei $\mathrm{Liu}^{1}$, and xiaoliang Shao ${ }^{1}$

${ }^{1}$ Affiliation not available

March 19, 2021

\begin{abstract}
Objective: The purpose of this study is to evaluate the changes of LA size and function by real-time 3D echocardiography (RT-3DE), and provide a better understanding about their effects on treatment and prognosis. Methods: Ten Bama miniature pigs were used for modeling STEMI. Images were obtained by RT-3DE at multiple time points. From the LA volume-time relation curve, the LA volume (LAV) and functions (storage, channel, and active emptying) at different phases were calculated. Blood samples were obtained for measurements of NT-proBNP, creatine kinase-MB(CK-MB) and cardiac Troponin-I(cTn-I). Results: (1) LAVmax, LAVmin and LAVpre-a were all increased with time. (2) The LA expansion index was the most sensitive index of LA functional change. It increased ( $\mathrm{p}<.05 \mathrm{vs}$. baseline) at the 1st day after STEMI (acute phase), and was correlated with diastolic function E/E' $(\mathrm{p}<.05)$, as shown by univariate correlation analysis. (3) On the 28th day after STEMI, the active emptying percentage of total emptying (AE) showed a continuously increasing trend $(\mathrm{p}<.05)$, and was correlated with VTILVOT (time-velocity integral of left ventricular outflow tract) $(\mathrm{p}<.05)$. Conclusions: During the acute phase after STEMI, LA volume changed in order to increase LV preload. After STIMI, the storage function of LA was the first to change, while active contractile function was significantly enhanced during subacute phase. With the accurate measurement of LA function with RT-3DE, our study will help improving the therapeutic target setting and pharmacologic interventions, which may enhance the clinical outcomes of STEMI patients.
\end{abstract}

\section{Assessment of Left Atrial Function after ST-Segment Elevation Acute Myocardial Infarction: An Application of Real Time Three-DimensionalEchocardiography}

Yuxia Miao, MD, $\mathrm{PhD}^{1}\left|\mathrm{Min} \mathrm{Xu}, \mathrm{MD}, \mathrm{PhD}^{1}\right|$ Fei Liu, MS, $\mathrm{PhD}^{1} \mid$ Yuetao Wang, MD, $\mathrm{PhD}^{2} \mid$ Xiaoliang $\mathrm{Shao}^{2}, \mathrm{MD}, \mathrm{PhD} \mid \mathrm{Xiao} \mathrm{Xie}, \mathrm{MD}, \mathrm{PhD}^{3}$

${ }^{1}$ Department of Echocardiography of The Third Affiliated Hospital of Soochow University, Chang Zhou, China;

${ }^{2}$ Department of Nuclear Medicine of The Third Affiliated Hospital of Soochow University, Chang Zhou , China

${ }^{3}$ Department of Ultrasonography of The Third Affiliated Hospital of Soochow University, Chang Zhou, China

Short Title : Assessment of Left Atrial Function after ST-Segment Elevation Acute Myocardial Infarction

\section{Correspondence}

Min Xu, MD, PhD, Department of Echocardiography, The Third Affiliated Hospital of Soochow University, Chang Zhou 213000, China.

E-mail: loisicelin@163.com 


\section{Tel: 13861058536}

Yuetao Wang, MD, PhD, Department of Nuclear Medicine, The Third Affiliated Hospital of Soochow University. Chang Zhou 213000, Jiangsu Province, China.

E-mail: yangjhsz1@163.com

Tel: 13401558906

Fei Liu and Xiao Xie contributed equally to this study

\section{KEYWORDS}

atrial function; left heart function; myocardial infarction; echocardiography; three-dimensional

Abbreviations: STEMI, ST-segment elevation myocardial infarction ; LA, left atrium; RT-3DE, realtime 3D echocardiography; LVEF , left ventricular ejection fraction; LAV, LA volume; CK-MB , creatine kinase-MB; cTn-I , cardiac Troponin-I; AE , active emptying percentage of total emptying; VTI , timevelocity integral; LVOT, left ventricular outflow tract; CHD, coronary heart disease; PCI, percutaneous coronary intervention; MI , myocardial infarct; LV, left ventricle; ACVA , American College of Veterinary Anesthesiologists; $\mathrm{LAV}_{\max }$, maximum LA volume ; $\mathrm{LAV}_{\min }$, minimum LA volume; $\mathrm{LAV}_{\text {pre-a }}$, LA volume before atrial active contraction.

\section{Abstract}

Objective: In ST-segment elevation myocardial infarction (STEMI), left atrial function exhibits superior predictive value over atrial size and left ventricular ejection fraction (LVEF). However, there is few studies about the dynamical changes of left atrial function after STEMI, as well as its correlation with left ventricular function. The purpose of this study is to evaluate the changes of LA size and function by real-time $3 \mathrm{D}$ echocardiography (RT-3DE), and provide a better understanding about their effects on treatment and prognosis.

Methods: Ten Bama miniature pigs were used for modeling STEMI. Images were obtained by RT-3DE at multiple time points: healthy state before surgery (baseline), $1^{\text {st }}$ day, $7^{\text {th }}$ days and $28^{\text {th }}$ day after STEMI. From the LA volume-time relation curve, the LA volume (LAV) and functions (storage, channel, and active emptying) at different phases were calculated. Blood samples were obtained for measurements of NTproBNP, creatine kinase-MB(CK-MB) and cardiac Troponin-I(cTn-I).

Results: (1) $\mathrm{LAV}_{\max }\left(p<.001\right.$ vs. baseline; $p<.05$ vs. $1^{\text {st }}$ day), $\operatorname{LAV}_{\min }(p<.001$ vs. baseline; $p<$. 05 vs. $1^{\text {st }}$ day) and $\operatorname{LAV}_{\text {pre-a }}\left(p<.001\right.$ vs. baseline $; p<.05$ vs. $7^{\text {th }}$ day) were all increased with time. $(2)$ The LA expansion index was the most sensitive index of LA functional change. It increased $(p<.05$ vs. baseline) at the $1^{\text {st }}$ day after STEMI (acute phase), and was correlated with diastolic function E/E' $(p<$ . 05), as shown by univariate correlation analysis. (3) On the $28^{\text {th }}$ day after STEMI, the active emptying percentage of total emptying (AE) showed a continuously increasing trend $(p<.05)$, and was correlated with $\operatorname{VTI}_{\text {LVOT }}$ (time-velocity integral of left ventricular outflow tract) $(p<.05)$.

Conclusions: During the acute phase after STEMI, LA volume changed in order to increase LV preload. After STIMI, the storage function of LA was the first to change, while active contractile function was significantly enhanced during subacute phase. With the accurate measurement of LA function with RT3DE, our study will help improving the therapeutic target setting and pharmacologic interventions, which may enhance the clinical outcomes of STEMI patients.

\section{1 | INTRODUCTION}

Coronary heart disease (CHD) is the leading cause of death and disability in China. For patients with acute ST-segment elevation myocardial infarction (STEMI), the most effective therapy for limiting myocardial infarct (MI) size is the timely myocardial reperfusion using either thrombolytic therapy or percutaneous 
coronary intervention (PCI). However, the morbidity and mortality of STEMI patients remain significantly high. Therefore, novel imaging technology and therapeutic interventions are required to improve the clinical outcomes of STEMI patients.

Studies have reported that the risk of death and stroke after STEMI increased with left atrium (LA) enlargement $^{1}$, highlighting the importance of conducting prospective studies to assess the LA functions of STEMI patients ${ }^{2,3}$. Atrial enlargement plays an important role in regulating LV filling, and thus, it may represent a comprehensive response to myocardial injury ${ }^{4}$. It has been shown that left atrial function exhibits superior predictive value over atrial size and left ventricular ejection fraction (LVEF) in STEMI ${ }^{5}$. However, there is currently no systematic study on LA function during acute and subacute phases of STEMI.

In order to quantify absolute and relative changes of LA function, we used an experimental pig model with induced ischemia and reperfusion. Each animal served as its own control. This approach allowed us to dynamically and continuously observe the immediate/early changes in atrial function and structure after STEMI, providing a special reference for non-invasive assessment of LA changes. Furthermore, advanced RT-3DE technique can stereoscopically improve the visualization of endocardial borders. When combined with the acquisition of time-volume curve, RT-3DE can significantly improve the LA volume calculation method $^{6}$. Studies have shown that the functional parameters acquired by left atrial time-volume curve are reliable and can be used to quantify storage, channel and active function ${ }^{7}$.

In this study, we used RE-3DE to record the volume and function of LA before surgery, at 1 day, 7 days, and 28 days after STEMI. We also analyzed the relationships between LA volume/function and the LV function at different time points. These results may be useful for improving the treatment intervention and clinical prognosis of STEMI patients.

\section{2 | MATERIAL AND METHODS}

\section{1 | Experimental Model}

We used male BA-MA miniature pigs (Guangxi) as the experimental animals. The pigs were 6 months old, weighing $22-25 \mathrm{~kg}$. As pigs can be very sensitive to stress, all pigs received adaptive feeding for one week to get used to personal conditions, location, and food. All pigs stayed in a barrier environment $\left(20-26^{\circ} \mathrm{C}\right)$ suitable for clean-grade experimental animals. The relative humidity of the environment was $40-70 \%$ and daily light time was 12-14 hours. The pigs were fed with a low-energy, high-fiber chow containing $16 \%$ protein, which followed the National standard for experimental animal full-price nutritional feed (GB14924-2001). The drinking water conformed to the requirements of GB 5749, up to aseptic conditions.

Before performing surgeries, the pigs were fasted for 12 hours. The multi-function monitor (Shenzhen Mindray Biomedical Electronics Co., Ltd,China) was used to continuously monitor blood pressure, heart rate and transcutaneous oxygen saturation. The anesthesia regimen was: $1.5 \mathrm{mg} / \mathrm{kg}$ diazepam and ketamine 25 $\mathrm{mg} / \mathrm{kg}$ to induce to induce anesthesia ${ }^{8}$. Propofol $2-4 \mathrm{mg} / \mathrm{kg} / \mathrm{h}$ was used to maintain anesthesia All anesthesia drugs were injected intravenously. After tracheal intubation, ventilator was used for assisted breathing ${ }^{9}$; bensulfonate atracurium and sufentanil were added in due course. We strictly followed the American College of Veterinary Anesthesiologists (ACVA) Guidelines (http://www.acva.org) for anesthesia monitoring. Anesthesia depth was assessed by a series of reflex signs, including palpebral and corneal reflexes, eye rotation, and degree of pupil dilation. Finally, at deep anesthesia, there was only slight or no local movement in response to a painful stimulus. Amiodarone $(150 \mathrm{mg} / 3 \mathrm{ml}$, Sanofi Winthrop Industrie, France) $75 \mathrm{mg} / \mathrm{h}$ was injected via i.v. to prevent malignant arrhythmia. After tracheal intubation, ventilator was used for assisted breathing. The electrocardiogram and blood pressure were monitored throughout the operation. The myocardial infarction modeling was performed under DSA via the balloon occlusion of coronary artery left anterior descending artery $(1 / 3$ to the middle of LAD)( Fig 1,a). Analgesia and anti-infection treatments were applied after operation. The animal was continuously monitored for several hours after extubation until the animal condition was stabilized. The electrocardiogram and hypersensitive troponin I were used to confirm the effectiveness of surgical modeling. This experiment conformed to the following guidelines: the guidelines of Animal Ethics Committee, the guidelines for care and use of laboratory animals published by 
the People's Republic of China (Document No. 55, 2001) and the National Institutes of Health of United States (NIH Publication No. 85-23, revised in 1996), and the revised Animals (Scientific Procedures) Act 1986 in the UK and Directive 2010/63/EU in Europe. The experiment was approved by the Animal Care and Use Committee of Soochow University.

\section{2 | Blood Sample Collection andMeasurements}

The ear vein blood of the pigs were collected after morning fasting. The collection method and quality control followed the recommendations of the European Society of Cardiology practical guidance ${ }^{10}$ and CardioOrmocheck study ${ }^{11}$. Two blood specimens were collected from each pig, and $2 \mathrm{ml}$ blood was stored in Ethylene Diamine Tetra-acetic Acid (EDTA) anticoagulation test tubes (Becton, Dickinson and Company, Franklin Lakes, NJ, USA) for NT-proBNP. After centrifugation at $3000 \mathrm{r} / \mathrm{min}$ for $10 \mathrm{~min}$, the supernatant was taken and processed with AU5800 automatic immunoassay instrument (Beckman Coulter, Brea, CA, USA) based on the Biosite NT-proBNP assay ${ }^{12}$. The lower detection limits was $10 \mathrm{pg} / \mathrm{ml}$. Another $2 \mathrm{~mL}$ blood sample was placed with separation gel in coagulant drying tube (non-anticoagulation tube), and the centrifuged serum was used for biochemical analysis. The levels of CK-MB and cTn-I were detected by enzyme method and immunoturbidimetry, respectively. The measurements ware completed by AU5800 automatic immunoassay instrument (Beckman Coulter, Brea, CA, USA), following the kit instructions.

\section{3 | Echocardiography}

Conventional and RT-3DE examinations (Epic 7C, Philips Healthcare Royal Philips Electronics, Netherlands) were performed on animals before surgery (baseline, healthy state), and at 1 day, 7 days and 28 days after modeling (STEMI). The 10 pigs were all photographed in left lateral position, connected with a 12-lead ECG; and the images were acquired at parasternal and apical views using a X5-1 (1 $5 \mathrm{MHz})$ probe with 15 $\mathrm{cm}$ in depth. The average frame rate was 50 frames/second. Standard Motion(M-), 2D and 3D images were obtained. All echocardiographic images were digitally stored, and the volume and functions were calculated by the commercially available QLAB (10.5; Philips) software package. Three cardiac cycles were continuously acquired from each section and averaged. The images were analyzed by two experienced doctors.

\section{4 | Parameters for LV Function and Hemodynamics}

In the apical four-chamber and two-chamber views, the end-diastolic and end-systolic volumes of LV, and the ejection fraction were calculated using biplane Simpson'S. In the apical four-chamber view, the pulsed Doppler was placed in the middle of the mitral valve tip to obtain the peak value $\mathrm{E}$ of early diastolic filling. The Doppler imaging of mitral ring was obtained in apical four-chamber view. The sampling volume of pulse Doppler was located on the septal side and the side wall of mitral ring, averaged by E', and the ratio E/E' was calculated as an indicator of LV filling pressure. The pulse Doppler of LV output tunnel (LVOT) at systolic phase was obtained from five-chamber heart view, and the velocity time integral (VTI) was then calculated.

\section{5 | Measuring and Calculation of LA Volume and Function}

The 3D image of LA was acquired during the respiration period using X5-1 (1 $5 \mathrm{MHz})$ probe in Full Volume mode. The average frame rate was 5 frames/second. The LA volume-time curve of the whole cardiac cycle was obtained by real-time 3D echocardiography, which yielded the measurements for maximum LA volume $\left(\mathrm{LAV}_{\max }\right)$, minimum LA volume $\left(\mathrm{LAV}_{\min }\right)$, and the $\mathrm{LA}$ volume before atrial active contraction $\left(\mathrm{LAV}_{\text {pre-a }}\right)$. $\mathrm{LAV}_{\text {pre-a }}$ was measured at the beginning of $\mathrm{P}$ wave in surface electrocardiogram(Fig $\left.2, \mathrm{a}-\mathrm{c}\right)$.

After the above measurements, the following LA emptying volume indices were calculated ${ }^{13-15}$ :

LA reservoir function was assessed using the following two indices:

1. expansion index $=\left[\left(\mathrm{LAV}_{\max }-\mathrm{LAV}_{\text {min }}\right) / \mathrm{LAV}_{\text {min }}\right] \times 100 \%$,

2. diastolic emptying index $=\left[\left(\mathrm{LAV}_{\max }-\mathrm{LAV}_{\min }\right) / \mathrm{LAV}_{\max }\right] \times 100 \%$.

LA conduit function was assessed using the following two indices: 
1. passive emptying percentage of total emptying $(\mathrm{PE})=\left[\left(\mathrm{LAV}_{\max }-\mathrm{LAV}_{\text {pre-a }}\right) /\left(\mathrm{LAV}_{\max }-\mathrm{LAV}_{\min }\right)\right] \times 100 \%$

2. passive emptying index $(\mathrm{PEI})=\left(\mathrm{LAV}_{\max }-\mathrm{LAV}_{\text {pre-a }}\right) / \mathrm{LAV}_{\max } \times 100 \%$.

LA booster pump function was assessed using the following two indices:

1. active emptying percentage of total emptying $(\mathrm{AE})=\left[\left(\mathrm{LAV}_{\text {pre-a }}-\mathrm{LAV}_{\min }\right) /\left(\mathrm{LAV}_{\max }-\mathrm{LAV}_{\min }\right)\right] \times 100 \%$

2. active emptying index $(\mathrm{AEI})=\left(\mathrm{LAV}_{\text {pre--a }}-\mathrm{LAV}_{\text {min }}\right) / \mathrm{LAV}_{\text {pre-a }} \times 100 \%$.

\section{6 | Statistical Analysis}

The measurement data that conformed to normal distribution were expressed as mean \pm standard deviation. Paired t test was used for comparison between the two groups, and ANOVA analysis was used for comparison between multiple groups. The measurement data with non-normal distribution was expressed by median (interquartile range), and the rank sum test was used for comparison between groups. Two-variable correlation analysis was performed using Spearman rank correlation. Multiple linear regression with stepwise variable selection were performed to determine predictors of LA functional changes, with which the significant univariate predictors were used as the model input. The variability between observers was evaluated by asking two independent observers to evaluate 10 randomly selected images. Each observer did not know the results from the other observer. The intra-observer variability was assessed by repeating measurements for 10 images by the same observer at 1 week after the first analysis. Bland-Altman analysis was performed to calculate the correlation coefficient and variable coefficient. $p<.05$ was considered statistically significant. SPSS 22.0 statistical software was used for data processing and analysis.

\section{3 | Results}

\section{1 | Angiography}

Coronary angiography showed that, after left anterior descending was completely occluded for 90 minutes, the collateral and distal vessels of the occluded artery did not restore blood supply(Fig 1,b-h). Electrocardiogram showed the typical dynamic changes of ST segment after anterior wall myocardial infarction (Fig 2,c), and the troponin I level was higher than normal. We observed sporadic ventricular premature beats in the STEMI pigs after successful modeling, but did not see atrial fibrillation.

\section{2 | All Echocardiographic and Biomarkers Measurements}

The STEMI modeling was successfully set up in all 10 pigs. For the representative pig, the LA volume-time curve was measured from one cardiac cycle during healthy state and at different time points after myocardial infarction. In order to exclude the influence of heart rate on cardiac cycle, the LA volume-time curve of the representative pig was obtained under standardized heart rate. The curve was expressed by LA volume/R-R interval (ml/s) (Fig 3). The baseline state and the echocardiographic features of 1 day, 7 days, and 28 days after modeling are summarized in TableI.

3.2.1 $\mathrm{LAV}_{\max }\left(p<.001\right.$ vs. baseline $; p<.05$ vs. $1^{\text {st }}$ day $), \operatorname{LAV}_{\min }\left(p<.001\right.$ vs. baseline $; p<.05$ vs. $1^{\text {st }}$ day) and $\mathrm{LAV}_{\text {pre-a }}\left(p<.001\right.$ vs. baseline; $p<.05$ vs. $7^{\text {th }}$ day) all gradually increased after STEMI. SV (stroke volume) increased on the first day after myocardial infarction $(p<.05)$, and was still increased at the $7^{\text {th }}$ day $\left(p<.01\right.$ vs. baseline); at the $28^{\text {th }}$ day after STEMI, the SV showed a decreasing trend $(p<.01$ vs. baseline). In addition, there were statistical differences in LVEF, $\mathrm{VTI}_{\mathrm{LVOT}}$, and E/E' at the four time points $(p<.01)$.

3.2.2 The expansion index increased at the ${ }^{\text {st }}$ day after STEMI ( $p<.05$ vs. baseline), and then decreased at the $28^{\text {th }}$ day $\left(p<.01\right.$ vs. $1^{\text {st }}$ day $; p<.05 \mathrm{vs.} 7^{\text {th }}$ day $)$. The AE decreased at the $1^{\text {st }}$ day after STEMI $(p>$ .05 vs. baseline), and then returned to baseline after the $7^{\text {th }}$ days and continued to increase at the $28^{\text {th }}$ day $\left(p<.05\right.$ vs. $7^{\text {th }}$ day; $p<.01$ vs. baseline). PE and PEI increased at the $1^{\text {st }}$ day after STEMI $(p>.05)$, and then fell below the baseline level at the $28^{\text {th }}$ day ( $p<.01$ vs. $1^{\text {st }}$ day; $p<.01$ vs. baseline). (Fig 4 ). 
3.2.3 Univariate correlation analysis showed that the expansion index of LA was associated with the diastolic function index E/E' $(p<.05)$ at the $1^{\text {st }}$ day after STEMI, there was no correlation between expansion index and CK-MB, cTn-I , NT-proBNP(Table II); On the $28^{\text {th }}$ day (subacute phase), the PE and PEI were both associated with left ventricular $\operatorname{VTI}_{\text {LVOT }}(p<.01)$. AE was also associated with $\mathrm{VTI}_{\text {LVOT }}$ at the $28^{\text {th }}$ day $(p<.05)$, there was no correlation between PE, PEI, AE and CK-MB, cTn-I, NT-proBNP(Table III).

\section{3 | Repeatability test}

The correlation coefficients between observers were 0.96 for $\mathrm{LAV}_{\max }$ and 0.94 for $\mathrm{LAV}_{\min }$; the variable coefficients were $6-11 \%$ for $\mathrm{LAV}_{\max }$ and $12-20 \%$ for $\mathrm{LAV}_{\min }$. The correlation coefficients within the observer were 0.97 for $\mathrm{LAV}_{\max }$ and 0.95 for $\mathrm{LAV}_{\text {min; }}$ the variable coefficients were $6-10 \%$ for $\mathrm{LAV}_{\max }$ and $13-22 \%$ for $\mathrm{LAV}_{\min }$. These data indicated good consistency between and within the observers (Fig 5).

\section{4 | DISCUSSION}

The main findings of the current study can be summarized as follows:(1) LA volume gradually increased over the 4 weeks period after STEMI. SV increased during the first 7days and then started to decrease; (2) LA expansion index was an important indicator for LA functional changes during the acute phase after STEMI. It can be used as a barometer for LV diastolic load; (3) During the subacute phase, LA active emptying function increased, which was independently associated with decreased LV hemodynamic parameters, indicating a compensatory response of LA. Meanwhile, LA channel function was a manifestation of decreased LV function. This study may help us understand the regulatory role of LA function in STEMI, furthermore, the clinical importance of atrial function may help improving the therapeutic target setting and pharmacologic interventions, which could enhance the clinical outcomes of STEMI patients.

Previous studies on STEMI patients showed that the LA enlarged immediately after myocardial infarction ${ }^{16}$, and the patients with LA remodeling experienced significantly worse progression in LA function during the follow-up ${ }^{17}$. The $\mathrm{LAV}_{\max }$ and $\mathrm{LAV}_{\min }$ both increased in our study $(p<.001)$. SV increased most significantly during the acute phase, which lasted for 7 days after myocardial infarction. The factors such as STEMI myocardial interstitial congestion, edema, increased stiffness of the ventricular wall, and decreased left ventricular diastolic capacity, can lead to increased left ventricular end-diastolic volume and pressure, increased resistance to blood flow from LA to LV, and obstructed left ventricular emptying in the late diastolic phase. According to Frank-starling's law, if the preload increases, the initial length of the left atrial muscle fibers will increase, and the LA volume will also increase. Therefore, the early expansion of LA during early myocardial infarction is a reliable marker for elevated LV pressure ${ }^{18}$. Ilic I et al ${ }^{19}$ studied the relationship between LA volume and pre-PCI ischemic time, and found that LA volume was a sensitive marker for increased LV filling pressure in STEMI patients. With the progression of myocardial infarction, the volume compensation function of LA reduced. As shown in our study, although LA volume continued to increase, SV showed a decreasing trend, indicating that LA couldn't increase the LV blood filling by enhancing SV; and thereby, SV was passively expanded. In this study, SV dynamically changed with myocardial infarction progression, indicative of the adaptive changes of LA volume to LV function. Kuhl JT et $\mathrm{al}^{20}$ have shown that the phased changes of LA volume during the cardiac cycle are associated with poor prognosis. Therefore, the measurements of LA volume after STEMI not only reflect the changes in cardiac function in real time, but also provide valuable prognostic information.

During the acute phase of STEMI, the function of LA is extremely important for LV filling. Because under the acute ischemic conditions in early phase, the LA reservoir function is strengthened to maintain a certain front load in order to keep normal LA filling ${ }^{17}$. Here, we showed that that the expansion index of reservoir period was a sensitive indicator for early atrial function changes during acute myocardial infarction which is related to the diastolic function index $\mathrm{E} / \mathrm{E}^{\prime}(p<.05)$. The diastolic function index $\mathrm{E} / \mathrm{E}$ ' is an important non-invasive indicator for evaluating LV filling pressure ${ }^{21}$, and is also an independent predictor for STEMI mortality ${ }^{22}$. Ischemic injury can result in diastolic dysfunction, which then leads to increased LA pressure. This is a compensation mechanism for LA to overcome the reduction of LV compliance ${ }^{23}$. Studies have shown that the diastolic dysfunction during acute myocardial infarction results in increased end-diastolic pressure, 
and the peak strain of LA reservoir phase is associated with LV end-diastolic pressure ${ }^{24}$, suggesting that LA reservoir peak strain can be used as a surrogate index for LV diastolic dysfunction ${ }^{25}$. LA distensibility was also an independent predictor of in-hospital mortality in 521 post-AMI patients $(\mathrm{HR}=2.37, p=0.026)^{26}$. Ersboll Mads et $\mathrm{al}^{27}$ measured LA function within 48 hours after STEMI, and showed that the LA function during reservoir phase was associated with death and prognosis of heart failure, indicating that the LA function during reservoir phase could serve as a standard for overall LA function and have potential use for pathophysiological diagnosis and prognosis. Dardas et $\mathrm{al}^{28}$ demonstrated that there was a significant association between LA function and LV diastolic dysfunction after extensive anterior myocardial infarction, and they were also related to increased cardiovascular disease and mortality. Therefore, in the acute phase of STEMI, the LA reservoir function is a sensitive indicator for changes in LA function. In the future, it is possible to improve the left ventricular diastolic function by adjusting the LA reservoir function through drugs or therapeutic interventions.

During the subacute phase of myocardial infarction, necrotic myocardium became fibrotic and the compensatory function of normal myocardium gradually disappeared, which led to decreased LV systolic function and reduced hemodynamic performance (as demonstrated by decreased $\mathrm{VTI}_{\mathrm{LVOT}}$ ). Doppler is a useful indicator for hemodynamics and can be used to calculate the myocardial mechanical index. This approach has become a standard method for assessing cardiac function ${ }^{29}$. Our study showed that the active contraction function of LA was significantly enhanced, while the LA passive function was reduced and was correlated with LV hemodynamic parameter $\mathrm{VTI}_{\mathrm{LVOT}}$. When the myocardial infarction entered subacute phase, the LA active emptying function was enhanced via sensing $\mathrm{VTI}_{\mathrm{LVOT}}$. Considering $\mathrm{LA}$ and LV structures as a whole, the growth of LA active systolic function seemed to act as a compensatory mechanism for decreased LV function. Our results were consistent with several previous studies: some studies found that among the 24 recruited patients with old anterior wall myocardial infarction, the increased LA active systolic function was associated with LV systolic dysfunction, indicating a compensatory response of $\mathrm{LA}^{25}$. Bozkurt et al ${ }^{30}$ followed 73 patients with anterior wall myocardial infarction who underwent PTCA; after one month, the active systolic function of LA was significantly increased $(p<.01)$, supporting the compensation of LA during left atrial remodeling. During the subacute phase, the increase in LV end-diastolic pressure and LA prefilled load, as well as the decrease in catheter volume, further led to the reduced early diastolic filling ${ }^{31}$. Therefore, the LA active systolic function can compensate for decreased LV function. Moreover, it may serve as a more sensitive parameter by providing the information on mechanical properties and functions of the LV myocardium. In addition, changes in LA active function might be a selective target to improve post-infarction function and negative left ventricular remodeling.

\section{1 | Limitations}

Several limitations should be addressed in our study. First, the sample size in this study is very limited due to the high cost of STEMI modelling in animal experiments. A large experiment or clinical research is necessary to further confirm the results. In this study, a model of myocardial infarction was constructed by occluding the coronary artery with balloon, but it may not completely imitate the way of STEMI occurred in the population. Only the immediate hemodynamic change was analyzed, the long-term hemodynamic changes and prognosis of STEMI should be included in future studies.

\section{5 | CONCLUSIONS}

Revascularization therapy is essential for STEMI. Recently, the improved understanding of pathophysiology of STEMI has led to a new observational perspective. In this study, we showed that during the acute phase of STEMI, reservoir function was a sensitive indicator for LA functional changes, and could be used as a barometer for LV diastolic load; during the subacute phase, the active contraction function of LA was enhanced, which was a compensation for the reduced left ventricular systolic function. As demonstrated by our study, RT-3DE provided a new approach to accurately and comprehensively evaluate cardiac functions in patients with myocardial infarction, offering a theoretical basis for assessing LA function. Moreover, our study suggests that improving left ventricular function by adjusting LA function may be a new approach to improve clinical outcomes in patients with STEMI. 


\section{Trial registration}

The study was approved by local Bioethics committee. This experiment followed the guidelines of Animal Ethics Committee and the guidelines for care and use of laboratory animals published by the People's Republic of China (Document No. 55, 2001) and the National Institutes of Health of United States (NIH Publication No. 85-23, revised in 1996). The experiment was also approved by the Animal Care and Use Committee of Soochow University.

\section{CONFLICT OF INTEREST}

The Authors declare that they have no conflict of interests.

\section{Funding information}

This work was supported by Natural Science Foundation of China (Grant NO.81471690) ; Application basic Project of Changzhou City Health Bureau (Grant NO.CJ20190086). Application basic Project of Changzhou City Health Bureau (Grant NO.WZ201804).

\section{Author Contributions}

Yuxia Miao, Min Xu, and Yuetao Wang participated in research design, data analysis and interpretation, and writing of the manuscript; Min Xu, Yuetao Wang, Fei Liu, and Xiao Xie participated in sample collection, and contributed to the acquisition and interpretation of data; Fei Liu, Xiao Xie, and Xiaoliang Shao participated in the execution of the experiments; Xiaoliang Shao participated in research design and supervised the course of the project.

\section{References}

1. Lehto M, Snapinn S, Dickstein K, et al. Prognostic risk of atrial fibrillation in acute myocardial infarction complicated by left ventricular dysfunction: the OPTIMAAL experience. Eur Heart J 2005;26(4):350-6.

2. Schmitt J, Duray G, Gersh BJ,et al. Atrial fibrillation in acute myocardial infarction: a systematic review of the incidence, clinical features and prognostic implications. Eur Heart J 2009;30(9):1038-45.

3. Avci E, Yildirim T, Aydin G,et al. Combining clinical predictors to better predict for the no-reflow phenomenon. European review for medical and pharmacological sciences 2018;22(15):4987-4994.

4. Trulock KM, Narayan SM, Piccini JP. Rhythm control in heart failure patients with atrial fibrillation: contemporary challenges including the role of ablation. J Am Coll Cardiol 2014;64(7):710-21.

5. Lonborg J T, Engstrom T, Moller J E, et al. Left atrial volume and function in patients following ST elevation myocardial infarction and the association with clinical outcome: a cardiovascular magnetic resonance study. European heart journal cardiovascular Imaging 2013;14(2):118-27.

6. Miyasaka Y, Tsujimoto S, Maeba H,et al. Left atrial volume by real-time three-dimensional echocardiography: validation by 64-slice multidetector computed tomography. J Am Soc Echocardiogr 2011;24(6):680-6.

7. Cameli M, Ciccone MM, Maiello M,et al.Speckle tracking analysis: a new tool for left atrial function analysis in systemic hypertension: an overview. J Cardiovasc Med (Hagerstown) 2016;17(5):339-43.

8. Kaiser GM, Fruhauf NR, Zhang H,et al.Intravenous infusion anesthesia with Propofol-Midazolam-fentanyl for experimental surgery in swine. J Invest Surg 2003;16(6):353-7.

9. Pehbock D, Dietrich H, Klima G, Paal P, Lindner KH, Wenzel V. Anesthesia in swine : optimizing a laboratory model to optimize translational research. Anaesthesist 2015;64(1):65-70.

10. Mueller C, McDonald K, de Boer RA,et al. Heart Failure Association of the European Society of Cardiology practical guidance on the use of natriuretic peptide concentrations. Eur J Heart Fail 2019;21(6):715-731. 
11. Prontera C, Zaninotto M, Giovannini S,et al. Proficiency testing project for brain natriuretic peptide (BNP) and the N-terminal part of the propeptide of BNP (NT-proBNP) immunoassays: the CardioOrmocheck study. Clin Chem Lab Med 2009;47(6):762-8.

12. Galderisi M, Lancellotti P, Donal E,et al.European multicentre validation study of the accuracy of E/e' ratio in estimating invasive left ventricular filling pressure: EURO-FILLING study. Eur Heart J Cardiovasc Imaging 2014;15(7):810-6.

13. Teo SG, Yang H, Chai P,et al. Impact of left ventricular diastolic dysfunction on left atrial volume and function: a volumetric analysis. Eur J Echocardiogr 2010;11(1):38-43.

14. Tsang TS, Barnes ME, Gersh BJ,et al.Left atrial volume as a morphophysiologic expression of left ventricular diastolic dysfunction and relation to cardiovascular risk burden. Am J Cardiol 2002;90(12):12849 .

15. Spencer KT, Mor-Avi V, Gorcsan J, et al. Effects of aging on left atrial reservoir, conduit, and booster pump function: a multi-institution acoustic quantification study. Heart 2001;85(3):272-7.

16. Thune JJ, Solomon SD. Left ventricular diastolic function following myocardial infarction. Curr Heart Fail Rep 2006;3(4):170-4.

17. Antoni ML, Ten Brinke EA, Marsan NA,et al.Comprehensive assessment of changes in left atrial volumes and function after ST-segment elevation acute myocardial infarction: role of two-dimensional speckle-tracking strain imaging. J Am Soc Echocardiogr 2011;24(10):1126-33.

18. Moller JE, Hillis GS, Oh JK,et al.Left atrial volume: a powerful predictor of survival after acute myocardial infarction. Circulation 2003;107(17):2207-12.

19. Ilic I, Stankovic I, Vidakovic R,et al.Relationship of ischemic times and left atrial volume and function in patients with ST-segment elevation myocardial infarction treated with primary percutaneous coronary intervention. Int J Cardiovasc Imaging 2015;31(4):709-16.

20. Kuhl JT, Moller JE, Kristensen TS,et al.Left atrial function and mortality in patients with NSTEMI an MDCT study. JACC Cardiovasc Imaging 2011;4(10):1080-7.

21. Ramkumar S, Yang H, Wang Y, et al. Association of the Active and Passive Components of Left Atrial Deformation with Left Ventricular Function. J Am Soc Echocardiogr 2017;30(7):659-666.

22. Hillis GS, Moller JE, Pellikka PA ,et al.Noninvasive estimation of left ventricular filling pressure by E/e' is a powerful predictor of survival after acute myocardial infarction. J Am Coll Cardiol 2004;43(3):360-7.

23. Abhayaratna WP, Seward JB, Appleton CP ,et al. Left atrial size: physiologic determinants and clinical applications. J Am Coll Cardiol 2006;47(12):2357-63.

24. Wakami K, Ohte N, Asada K,et al. Correlation between left ventricular end-diastolic pressure and peak left atrial wall strain during left ventricular systole. J Am Soc Echocardiogr 2009;22(7):847-51.

25. Yurdakul S, Aytekin S. Left atrial mechanical functions in patients with anterior myocardial infarction: a velocity vector imaging-based study. Kardiol Pol 2013;71(12):1266-72.

26. Hsiao SH, Chiou KR, Porter TR, et al. Left atrial parameters in the estimation of left ventricular filling pressure and prognosis in patients with acute coronary syndrome. Am J Cardiol 2011;107(8):1117-24.

27. Ersboll M, Andersen MJ, Valeur N ,et al.The prognostic value of left atrial peak reservoir strain in acute myocardial infarction is dependent on left ventricular longitudinal function and left atrial size. Circ Cardiovasc Imaging 2013;6(1):26-33.

28. Dardas PS, Pitsis AA, Mezilis NE ,et al.Left atrial function and work after surgical ventricular restoration in postmyocardial infarction heart failure. J Am Soc Echocardiogr 2008;21(7):841-7. 
29. Yao GH, Zhang M, Yin LX, et al. Doppler Echocardiographic Measurements in Normal Chinese Adults (EMINCA): a prospective, nationwide, and multicentre study. Eur Heart J Cardiovasc Imaging 2016;17(5):512-22.

30. Bozkurt E, Arslan S, Acikel M, et al.Left atrial remodeling in acute anterior myocardial infarction. Echocardiography 2007;24(3):243-51.

31. Boyd AC, Ng AC, Tran da T, et al.Left atrial enlargement and phasic function in patients following non-ST elevation myocardial infarction. J Am Soc Echocardiogr 2010;23(12):1251-8.

\section{Figure legends}

FIG 1 Induction of myocardial infarction via percutaneous balloon occlusion of LAD. Coronary angiography performed in the anteroposterior view before (a) and after (b) balloon inflation with confirmation of distal occlusion immediately after the second diagonal branch of the mid-LAD (red arrow). Trimethyl tetrazolium chloride stained the myocardium of left ventricular papillary muscle section (c) and apical section (d) . Infarcted myocardium appears in white (red arrow) while normal myocardium appears in red in the slice. Pathological analysis, (e)showing the normal left ventricular myocardial tissue stained by HE. (f) shows the left ventricular myocardial infarction area tissue stained by HE. (g) Showing the normal left ventricular myocardial tissue stained by pathological MASSON. (h) shows the myocardial infarction area of the left ventricle anterior wall stained by MASSON. LAD, descend forward left.

FIG 2 (a) Twelve lead electrocardiogram after balloon inflation at 60 minutes, exhibiting significant STsegment elevation in leads of V1-V4 with reciprocal ST segment depressions in II, III and aVF. Real-time three-dimensional echocardiography to obtain LAVmax(b), LAVpre-a(c) and LAVmin(d).

FIG 3 .The averaged LA volume waveforms at each time point. $\mathrm{X}$ axis denotes the time during one cardiac cycle. To adjust differences in heart rate, we divided the LA volume by the time of each cardiac cycle. Y axis denotes the LA volumes in each group. AVC, Aortic valve closure.

FIG 4 .The temporal changes of LA functional parameters following STEMI. (a), LA reservoir function : expansion index $(*)$, diastolic emptying index ();(b), LA conduit function :PE $(*)$, PEI (); (c),LA booster pump function: $\operatorname{AE}(*)$, AEI ()$;(d), \operatorname{LVEF}(*)$. AE, active emptying percentage of total emptying; AEI, active emptying index; PE, passive emptying percentage of total emptying; PEI, passive emptying index, LVEF, left ventricular ejection fraction.

FIG 5 . Intraobserver and Interobserver Variability. The Bland-Altman plots show the intraobserver (a, b ) and interobserver $(\mathbf{c}, \mathbf{d})$ variability for measuring $\mathrm{LAV}_{\max }$ and $\mathrm{LAV}_{\min }$. The solid line indicates the mean value of the difference, and the dashed line indicates mean 1.96 SD.

TABLE 1 . Volume, functional and biochemical parameters before and after STEMI

\begin{tabular}{|c|c|c|c|c|}
\hline & Baseline $(n=10)$ & $1 \operatorname{day}(\mathrm{n}=10)$ & $7 \operatorname{day}(\mathrm{n}=10)$ & $28 \operatorname{day}(\mathrm{n}=10)$ \\
\hline$\overline{\mathrm{LAVmax}(\mathrm{ml})}$ & $26.9 \pm 7.4$ & $33.6 \pm 8.6$ & $38.3 \pm 7.7^{* *}$ & $45.1 \pm 7.6^{* *}$ \\
\hline $\operatorname{LAVmin}(\mathrm{ml})$ & $16.5 \pm 5.0$ & $18.2 \pm 8.9$ & $19.8 \pm 4.3$ & $28.2 \pm 5.3^{* *}, \# \#$ \\
\hline LAV pre-a(ml) & $21.6 \pm 5.3$ & $25.2 \pm 7.2$ & $30.2 \pm 7.9^{* *}$ & $41.0 \pm 7.6^{* *}, " \# \#$ \\
\hline $\mathrm{SV}(\mathrm{ml})$ & $10.5 \pm 2.6$ & $15.4 \pm 7.5^{* *}$ & $18.5 \pm 5.4^{* *}$ & $16.9 \pm 4.0^{* *}$ \\
\hline Expansion index $(\%)$ & $65.5 \pm 12.0$ & $111.9 \pm 68.0^{*}$ & $97.1 \pm 32.6$ & $61.2 \pm 15.5, \#$ \\
\hline Diastolic emptying index(\%) & $39.3 \pm 4.3$ & $47.0 \pm 20.6$ & $48.0 \pm 8.9$ & $37.4 \pm 6.0$ \\
\hline $\mathrm{PE}(\%)$ & $48.9 \pm 16.6$ & $56.5 \pm 17.7$ & $42.4 \pm 19.0$ & $24.5 \pm 9.1^{* *}$, ,\#\# \\
\hline PEI $(\%)$ & $19.3 \pm 7.2$ & $25.1 \pm 12.1$ & $21.4 \pm 12.1$ & $9.3 \pm 4.0^{*}, \# \#$ \\
\hline $\mathrm{AE}(\%)$ & $51.1 \pm 16.6$ & $43.5 \pm 17.7$ & $57.6 \pm 19.0$ & $75.5 \pm 9.1^{* *}, \# \#$ \\
\hline AEI (\%) & $24.4 \pm 6.6$ & $30.1 \pm 19.6$ & $33.6 \pm 6.8$ & $31.0 \pm 5.6$ \\
\hline LVEF (\%) & $67.4 \pm 4.8$ & $54.1 \pm 4.9^{* *}$ & $49.9 \pm 5.9^{* *}$ & $49.3 \pm 3.4^{* *}$ \\
\hline $\mathrm{E}(\mathrm{cm} / \mathrm{s})$ & $70.0 \pm 11.4$ & $64.5 \pm 15.9$ & $69.1 \pm 18.1$ & $69.3 \pm 11.8$ \\
\hline
\end{tabular}




\begin{tabular}{lllll}
\hline & Baseline $(\mathrm{n}=10)$ & 1 day $(\mathrm{n}=10)$ & 7 day $(\mathrm{n}=10)$ & 28 day $(\mathrm{n}=10)$ \\
\hline $\mathrm{A}(\mathrm{cm} / \mathrm{s})$ & $49.6 \pm 8.9$ & $46.2 \pm 9.3$ & $52.6 \pm 8.0$ & $52.2 \pm 8.8$ \\
$\mathrm{E} / \mathrm{A}$ & $1.4 \pm 0.2$ & $1.4 \pm 0.3$ & $1.3 \pm 0.3$ & $1.3 \pm 0.2$ \\
$\mathrm{E} / \mathrm{E}$ & $6.4 \pm 2.8$ & $8.3 \pm 4.0$ & $5.3 \pm 1.4^{\#}$ & $5.6 \pm 1.9$ \\
$\mathrm{LVEDV}(\mathrm{ml})$ & $39.3 \pm 6.9$ & $48.6 \pm 21.6$ & $53.9 \pm 11.6^{*}$, & $67.7 \pm 16.9^{*}, \#$ \\
VTI $_{\text {LVOT }}(\mathrm{cm})$ & $21.4 \pm 4.1$ & $15.8 \pm 2.9^{* *}$ & $17.3 \pm 3.6^{*}$ & $18.2 \pm 2.8^{*}$ \\
NT-proBNP$(\mathrm{ng} / \mathrm{l})$ & $145.5(111.3,173.9)$ & $1965.0(934.0,2560.0)^{* *}$ & $755.7(546.8,1197.0)^{* *}$ & $938.8(627.7,1546.0)$ \\
CK-MB $(\mathrm{ng} / \mathrm{ml})$ & $0.30(0.18,0.40)$ & $115.0(103.8,135.8)^{* *}$ & $0.40(0.28,0.50)$ & $0.40(0.08,0.63)$ \\
Troponin-I $(\mathrm{ng} / \mathrm{ml})$ & $0(0,0.04)$ & $39.85(18.03,51.60)^{* *}$ & $2.85(2.03,4.05)$ & $0.04(0.02,0.04)$ \\
\hline
\end{tabular}

Abbreviations: SV, stroke volume; AE, active emptying percentage of total emptying; AEI, active emptying index; PE, passive emptying percentage of total emptying; PEI , passive emptying index; SV, stroke volume ; LVEDV, left ventricular end diastolic volume; E, the early mitral diastolic flow peak value; A, late mitral diastolic velocity; E/E', the early mitral diastolic flow peak value(E)/mitral tissue doppler early diastolic peak value(E'); $\mathrm{VTI}_{\mathrm{LVOT}}$, the velocity time integral of $\mathrm{LV}$ outflow tract; $\mathrm{LVEF}$, left ventricular ejection fractions.

${ }^{*} p<0.05$ versus baseline; ${ }^{* *} p<0.01$ versus baseline.

$p<0.05$ versus $1^{\text {st }}$ day; $p<0.01$ versus $1^{\text {st }}$ day.

${ }^{\#} p<0.05$ versus $7^{\text {th }}$ day; ${ }^{\# \#} p<0.01$ versus $7^{\text {th }}$ day.

Table 2. Univariate correlations with LA functional parameter and LV echocardiography parameters at 1st day after STEMI

\begin{tabular}{lll}
\hline & Expansion index & Expansion index \\
\hline & $\mathbf{R}$ & $\mathrm{p}$ \\
$\mathrm{LVEF}(\%)$ & -0.204 & 0.571 \\
$\mathrm{E}(\mathrm{cm} / \mathrm{s})$ & -0.296 & 0.406 \\
$\mathrm{~A}(\mathrm{~cm} / \mathrm{s}$ & -0.409 & 0.241 \\
E/A & 0.305 & 0.391 \\
E/E' & -0.667 & $0.031^{*}$ \\
LVEDV $(\mathrm{ml})$ & -0.653 & 0.063 \\
VTI $_{\mathrm{LVOT}}(\mathrm{cm})$ & -0.444 & 0.204 \\
NT-proBNP(ng/l) & -0.479 & 0.166 \\
CK-MB $(\mathrm{ng} / \mathrm{ml})$ & -0.006 & 0.998 \\
Troponin-I(ng/ml) & -0.346 & 0.330 \\
\hline
\end{tabular}

Abbreviations: LVEF, left ventricular ejection fractions; E, the early mitral diastolic flow peak value; A, late mitral diastolic velocity ;E/E', the early mitral diastolic flow peak value(E)/mitral tissue doppler early diastolic peak value(E'); LVEDV, left ventricular end diastolic volume; $\mathrm{VTI}_{\mathrm{LVOT}}$, the velocity time integral of LV outflow tract.

${ }^{*} p<.05$.

Table 3 . Univariate correlations with LA functional parameters and LV echocardiography parameters at the 28th day after STEMI 


\begin{tabular}{|c|c|c|}
\hline Parameter & $\mathbf{R}$ & $\mathbf{p}$ \\
\hline & Passive emptying percentage of total emptying(PE) & Passive emptying percentage of tot \\
\hline $\operatorname{LVEF}(\%)$ & 然 & 0.551 \\
\hline $\mathrm{E}(\mathrm{cm} / \mathrm{s})$ & -0.114 & 0.754 \\
\hline $\mathrm{A}(\mathrm{cm} / \mathrm{s}$ & -0.025 & 0.945 \\
\hline $\mathrm{E} / \mathrm{A}$ & -0.147 & 0.686 \\
\hline $\mathrm{E} / \mathrm{E}^{\prime}$ & -0.156 & 0.692 \\
\hline LVEDV(ml) & -0.365 & 0.311 \\
\hline $\operatorname{VTI}_{\mathrm{LVOT}}(\mathrm{cm})$ & 0.843 & $<0.01^{* *}$ \\
\hline NT-proBNP(ng/l) & -0.347 & 0.324 \\
\hline $\mathrm{CK}-\mathrm{MB}(\mathrm{ng} / \mathrm{ml})$ & 0.006 & 0.989 \\
\hline Troponin-I(ng/ml) & -0.085 & 0.814 \\
\hline
\end{tabular}

Footnote: LVEF: left ventricular ejection fractions; E, the early mitral diastolic flow peak value; A, late mitral diastolic velocity ;E/E',early mitral diastolic flow peak value(E)/mitral tissue doppler early diastolic peak value(E'); LVEDV, left ventricular end diastolic volume; $\mathrm{VTI}_{\mathrm{LVOT}}$, the velocity time integral of $\mathrm{LV}$ outflow tract.

${ }^{* *} p<.01$.

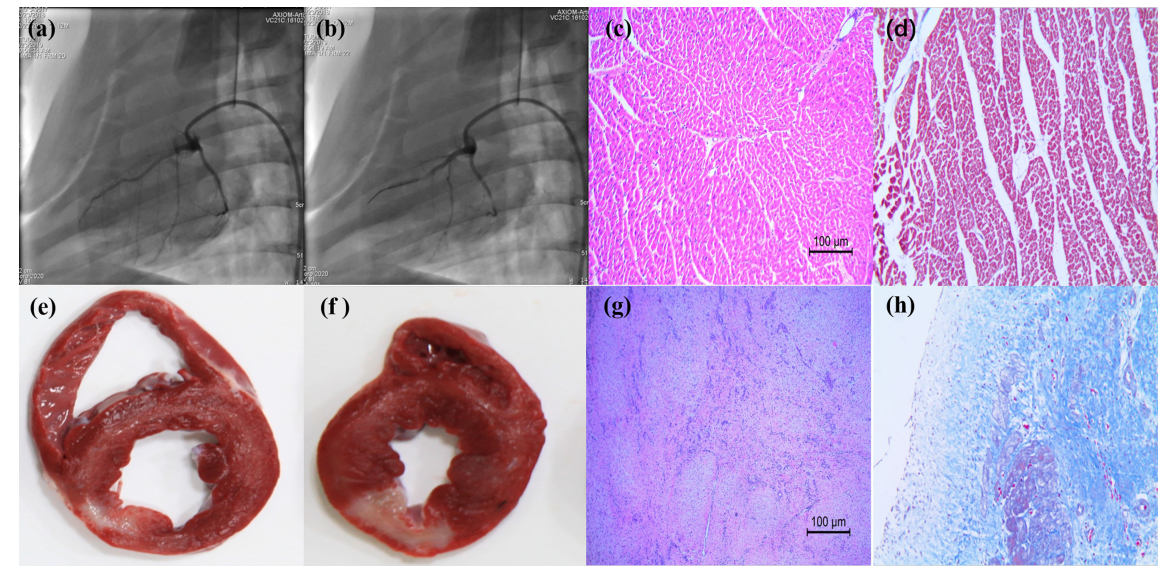



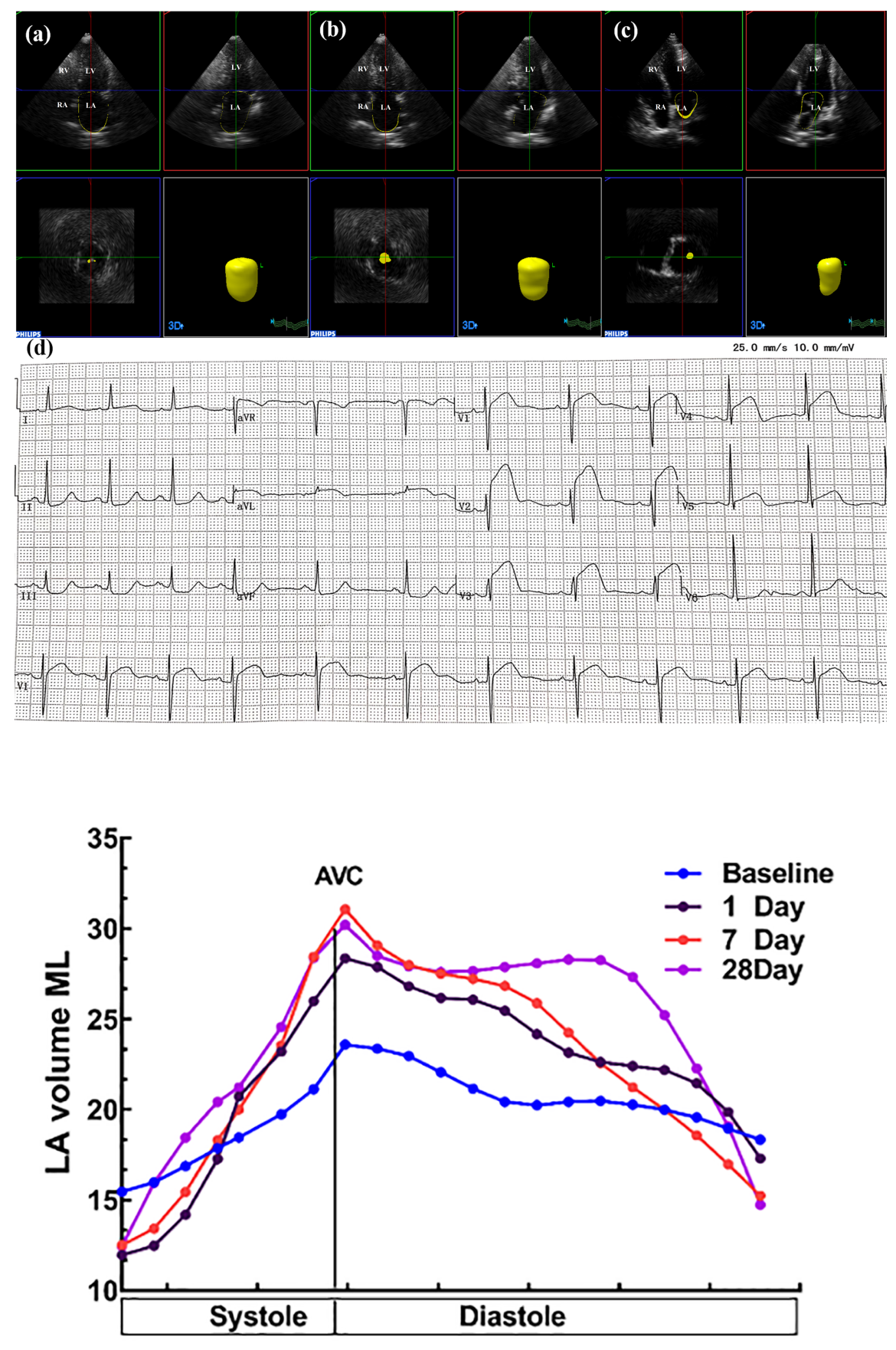

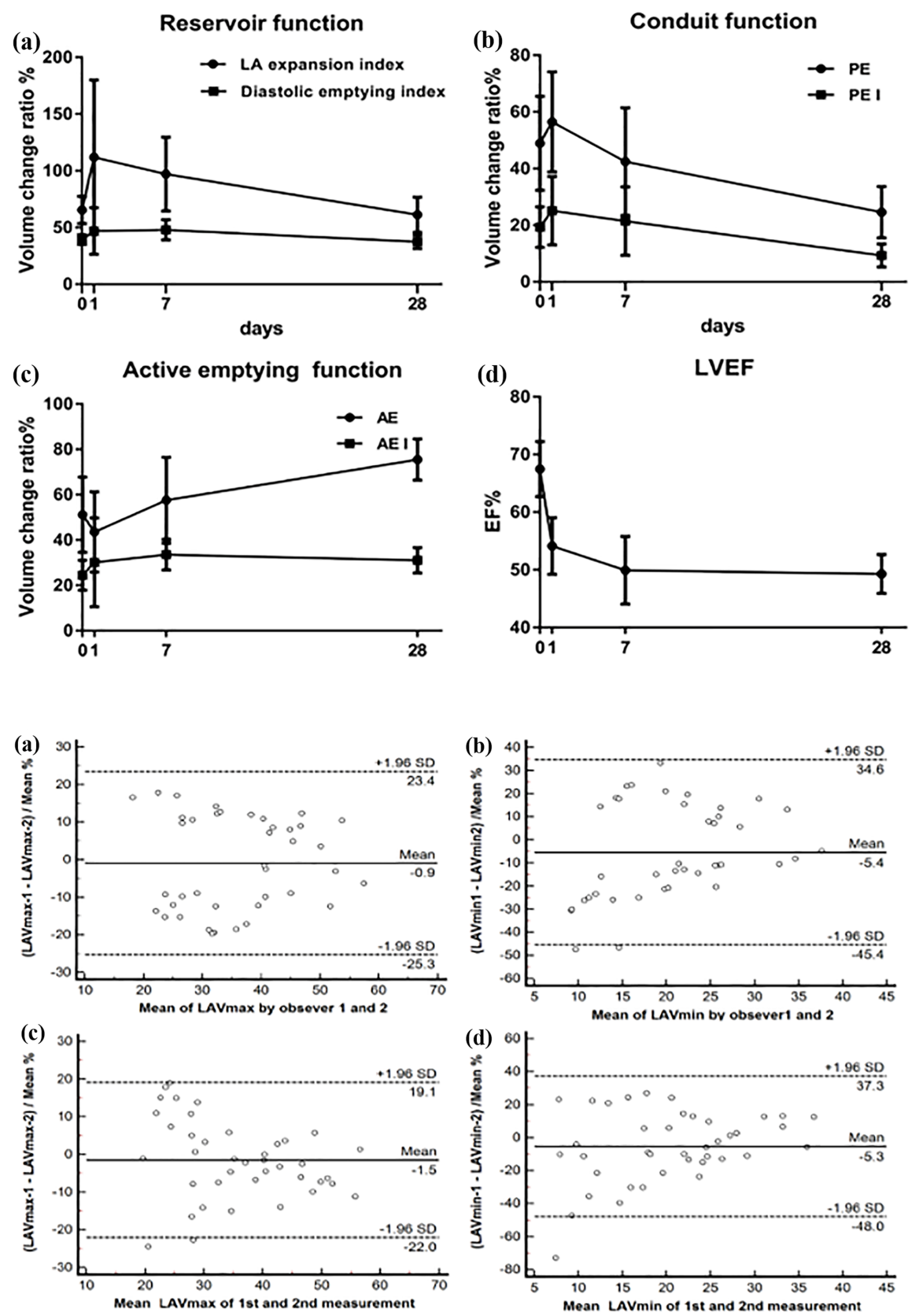